\title{
Enhancements to Threshold Based Range Estimation for Ultra-Wideband Systems
}

\author{
Hamza Soganci*, ${ }^{*}$, Sinan Gezici*, and Mehmet Burak Guldogan ${ }^{\sharp}$ \\ * Department of Electrical and Electronics Engineering, Bilkent University, 06800, Ankara, Turkey \\ $\natural$ Group of Electronic Systems and Flight Disciplines, TUBITAK SAGE, 06261, Ankara, Turkey \\ $\sharp$ Department of Electrical and Electronics Engineering, Turgut Ozal University, 06010, Ankara, Turkey \\ Emails: hsoganci@ee.bilkent.edu.tr,gezici@ee.bilkent.edu.tr, bguldogan@turgutozal.edu.tr
}

\begin{abstract}
Ultra-wideband (UWB) signals have very high time resolution, which makes them a very good candidate for range estimation based wireless positioning. Although the accuracy is the major concern for range estimation, it is also important to have low-complexity algorithms that can be employed in real time. In this study, two low-complexity range estimation algorithms are proposed for UWB signals, which achieve improved performance compared to the state-of-the-art low-complexity ranging algorithms. The proposed algorithms are inspired from two well-known algorithms; 'serial backward search' (SBS) and 'jump back and search forward' (JBSF). Performances of the proposed algorithms are compared with those of the SBS and JBSF algorithms based on real measurements. In addition, theoretical bounds are calculated in order to quantify the statistical performance of the algorithms.
\end{abstract}

Keywords: Ultra-wideband, range estimation, wireless positioning, Cramér-Rao lower bound.

\section{INTRODUCTION}

Ultra-wideband (UWB) signals are characterized by their very large bandwidths compared to those of conventional narrowband and wideband signals [1], [2]. Federal Communications Commission (FCC) defines a UWB signal to have an absolute bandwidth of at least $500 \mathrm{MHz}$ or a fractional (relative) bandwidth of larger than 20\% [3]. Due to the high absolute bandwidth, UWB signals can be realized by very short duration waveforms, which are well-suited for accurate range and position estimation. In addition, UWB signals have high penetration capability through the obstacles because of their high relative bandwidth [4]. As a result, these signals can be used for range and position estimation not only in line-ofsight (LOS) situations but also in non-line-of-sight (NLOS) scenarios.

Due to its unique characteristics, the UWB technology can be used in short range wireless sensor network (WSN) applications for which accurate range and position estimation is critical [5]. In particular, UWB signals can be employed in many different applications such as surveillance of high security areas, monitoring critical body activities of a patient, inbuilding robot guidance, automated handling, and see-throughthe-wall radar imaging for rescue/military [4], [6].

The main focus of this study is to develop low-complexity and robust range estimation algorithms for UWB systems.

\footnotetext{
${ }^{0}$ This work was supported in part by Turk Telekom (project no. 3015-02) and in part by the European Commission in the framework of the FP7 Network of Excellence in Wireless Communications (contract no. 318306).
}

Low-complexity UWB range estimation algorithms are desirable for applications with strict power and computational complexity constraints. Among such algorithms is the 'ranging with largest- $N$ peak-detection', which considers the largest $N$ energy samples (or, correlation peaks) and estimates the timeof-arrival (equivalently, the range) based on those samples [7]. In particular, the range is estimated according to the energy sample that has the minimum time index among the largest $N$ energy samples. There exist different versions of this approach in order to achieve accurate range estimation in various scenarios [7]. Another low-complexity UWB range estimation algorithm is 'serial backward search' (SBS), which estimates the range by searching energy samples in the backward direction (i.e., in the direction of decreasing time indices) starting from the strongest energy sample [8]. A different approach for low-complexity UWB range estimation is the 'jump back and search forward' (JBSF) algorithm. In this approach, the strongest energy sample is detected first and then the energy samples are searched in the forward direction starting from an energy sample that is before the strongest one by a certain number of samples. The search continues until an energy sample exceeds the specified threshold [4], [8]. As investigated in various studies such as [9], the SBS and JBSF algorithms can provide accurate range estimation for UWB systems in real environments.

In this study, we propose two UWB range estimation algorithms, which are inspired from the SBS and JBSF algorithms. The first one is a modified version of the SBS algorithm, which provides a more robust version via performing multiple threshold comparisons. The second one is similar to the JBSF algorithm but employs a completely different test for determining the index of the first arriving signal path. The proposed algorithms are tested on real data collected from an office environment. In addition, comparisons against the JBSF and SBS algorithms, and the Cramér-Rao lower bound (CRLB) are presented. The main contribution of this study is that two lowcomplexity UWB range estimation algorithms are proposed, which provide more accurate and robust range estimation than the state-of-the-art algorithms. In addition, performance evaluation is performed on real UWB measurements and also the CRLB comparisons are provided.

The remainder of the paper is organized as follows: First brief information about threshold based range estimation algorithms is presented. After that, two new threshold based range estimation algorithms are proposed. Then, performances of the proposed algorithms are compared with the existing techniques based on real measurements. Finally, theoretical limits for this 


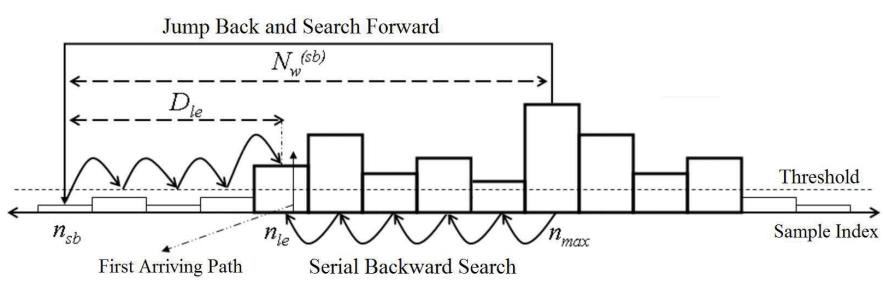

Fig. 1. Illustration of JBSF and SBS algorithms. Here $n_{\max }$ is the index of the strongest sample, $n_{l e}$ is the index of the first arriving path, $N_{w}(s b)$ is the number of samples to be jumped back, $n_{s b}$ is the index of the sample where the forward search begins and $D_{l e}$ is the delay between the first sample of the search window and the first arriving path [8].

problem are provided to statistically quantify the performance of the algorithms.

\section{THRESHOLD BASED UWB RANGE ESTIMATION}

Large bandwidths of UWB signals result in very high time resolution, which facilitates accurate time-of-arrival, hence range, estimation. Although various UWB range estimation algorithms are available in the literature [1], many of them do not present favorable trade-offs between accuracy and complexity. For example, maximum likelihood (ML) based estimators provide very accurate results. However, in the case of no prior information about time delays and channel coefficients of multipath components, all these parameters should be jointly estimated in calculating the ML estimate [10]. This creates a huge computational load and makes it almost impossible to operate in real time. Therefore in practical applications ML based estimators are not commonly preferred.

Since the computational complexity is a primary concern for practical applications, several low-complexity UWB range estimators are proposed in the literature [10]. These estimators try to find the time index of the first arriving path, $n_{l e}$, in the received signal. Then based on this index, the range is estimated as follows:

$$
\hat{r}=c\left(n_{l e} T_{s}+\frac{T_{s}}{2}\right),
$$

where $c$ denotes the speed of light and $T_{s}$ denotes the sampling time (period). (If a two-way ranging protocol [11] is employed, then the range is estimated by dividing the expression in (1) by two.) Depending on how they estimate the index of the first arriving path $n_{l e}$, there exist various low-complexity range estimators. Although performance of these estimators is limited compared to ML based estimators, some of them can still offer satisfactory accuracy alongside low computational complexity. An important class of such estimators is the threshold based range estimators. These estimators determine the first path component of the received signal by comparing the (energy) samples of the received signal against a certain threshold [8], [12], [13].

JBSF and SBS algorithms are two well-known examples of threshold based range estimators [8]. These algorithms are illustrated in Fig. 1. Both algorithms start by finding the strongest sample in the received signal. After finding the strongest sample, SBS performs a backward search starting from that sample and compares each sample with a certain threshold. It selects the first sample that is above the threshold which is followed in the search direction by a sample that is below the threshold [10]. This approach is problematic when there are many noise-only samples between the first arriving path and strong multipath components. In that case the algorithm may terminate early and never reach to the first arriving path. On the other hand, after finding the strongest sample, JBSF jumps a certain number of samples back from that sample and performs a forward search. It compares each sample with a certain threshold and selects the first sample that is above the threshold [8]. This approach may fail when the strong multipath components are far away from the first arriving path. In that case the algorithm may jump to a sample coming after the first arriving path and never have a chance to reach to the sample coming from the first arriving path. The intuition behind both algorithms is that as the first signal path commonly resides before the strongest signal sample, it is useful to perform a search among the samples coming before the strongest one [10]. Both algorithms perform well under certain conditions but they are still prone to making errors since both algorithms make the decision by considering just one sample that is above or below the threshold.

\section{PROPOSED TECHNIQUES}

JBSF and SBS are similar in the sense that they both choose the first sample that is above or below a certain threshold in their search directions. The problem with this approach is that the samples coming after that specific sample are not considered at all, and making a decision based on just one sample is not always reliable. Therefore, the main motivation behind the proposed approaches in this section is to take multiple samples into account in order to make more reliable decisions and provide robustness.

As discussed in Section II, the SBS algorithm finds the largest sample in the received signal first and then compares the values of the previous samples with a threshold one by one. Then it considers the first sample which is below that threshold. Obviously, having one sample below the threshold does not ensure that the samples coming before that sample are also below the threshold; i.e., in the noise-only region. Therefore, to ensure the reliability of the estimation, we need to keep on searching even one sample below the threshold is found. The proposed modification to SBS is to continue to search until a specific number of consecutive samples that are below the threshold are detected.

Before describing the proposed algorithm, called modified $S B S$, in more detail, the transmitted signal model for a UWB system is provided first:

$$
x(t)=\sum_{j=0}^{N_{f}-1} \sum_{i=1}^{N_{p}} p\left(t-i T_{p}-j T_{f}\right),
$$

where $p(t)$ represents the UWB pulse, $T_{p}$ is the pulse duration, $T_{f}$ is the duration of a frame, $N_{p}$ is the number of pulses in a frame, and $N_{f}$ is the number of frames. The signal in (2) is sent from a UWB transmitter, and the received signal is processed in order to estimate the time-of-arrival of the incoming signal, which is obtained by estimating the time index of the first arriving signal path. 


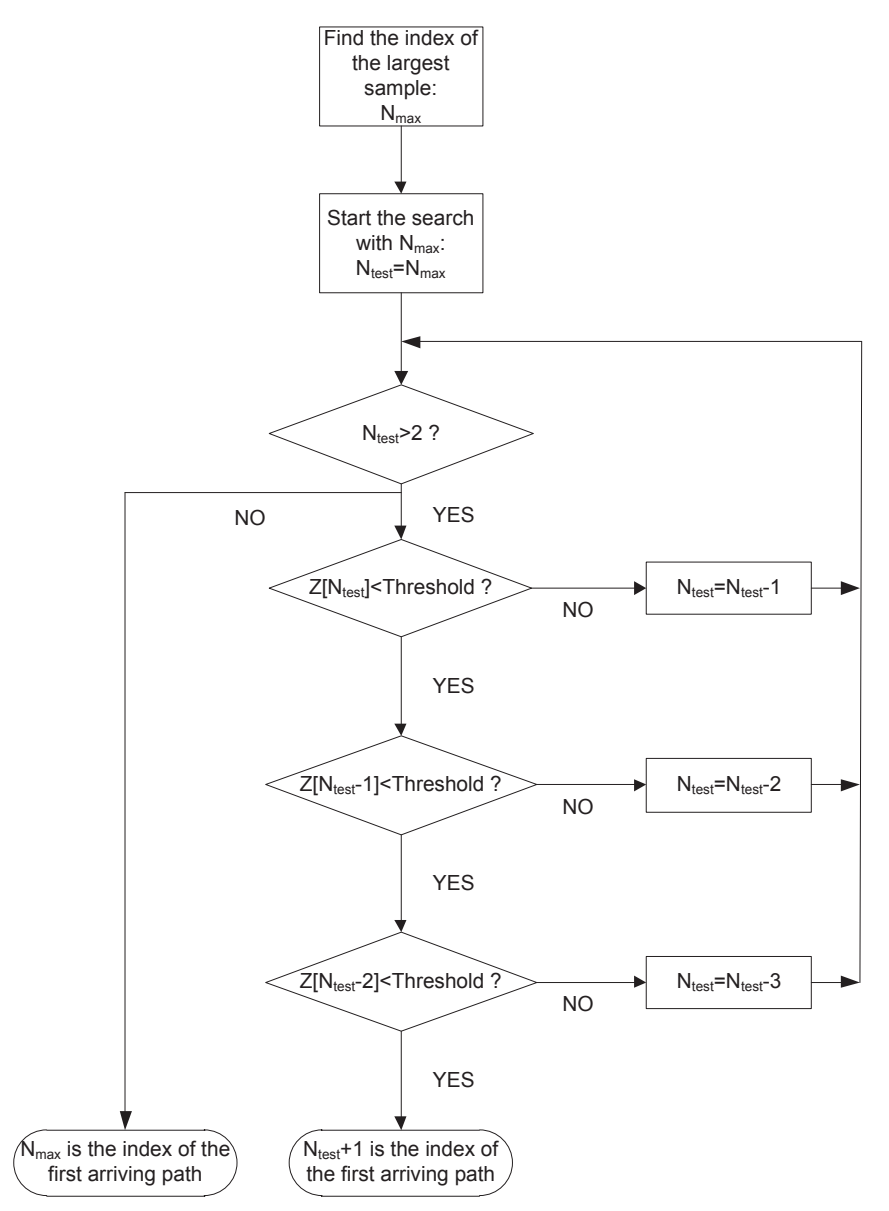

Fig. 2. Flowchart of the modified SBS algorithm.

Let $z[k]$ represent the samples of an energy detector output at the receiver (or, absolute values of the samples of a correlator output) for $k=1, \ldots, N_{b}$. The modified SBS algorithm starts by finding the index of the largest sample, which is the first sample to be compared with the threshold:

$$
N_{\text {test }}=N_{\text {max }}=\underset{k \in\left[1, N_{b}\right]}{\arg \max } z[k] .
$$

Then, the algorithm compares the values of the previous samples with the threshold and searches for a certain number of consecutive samples that are all below the threshold. If the algorithm finds such a set of consecutive samples starting from $N$, then $N+1$ is declared as the index of the first arriving path. Otherwise $N_{\max }$ is decided to be the index of the first arriving path. The flowchart of the modified SBS algorithm searching for three consecutive samples is shown in Fig. 2. It is noted that the number of consecutive samples to be tested is an important design parameter that should be selected according to the signal and channel characteristics. As discussed in Section V, it is observed via experiments that testing two or three consecutive samples provides the best results in most cases.

The second proposed algorithm is called modified JBSF. As mentioned in the previous section, the standard JBSF algorithm finds the largest sample in the received signal first and then jumps back to a specific sample and compares the values of

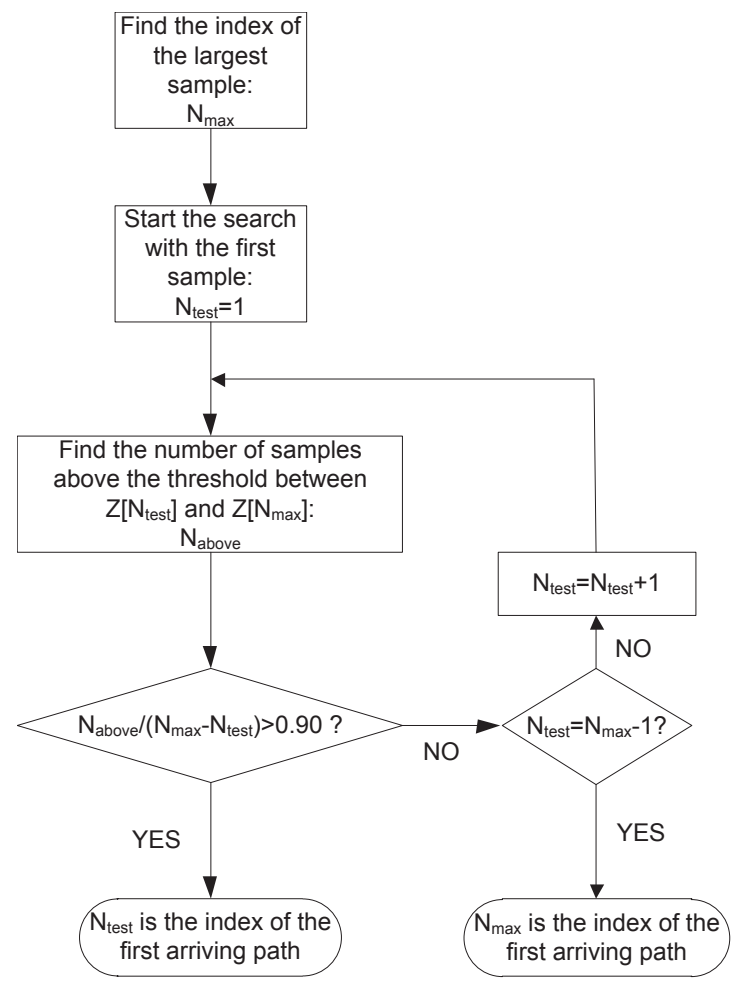

Fig. 3. Flowchart of the modified JBSF algorithm.

the following samples with a threshold one by one. Then the first sample which is above that threshold is selected by the algorithm. However, it is possible that this sample can be a pure noise sample which is followed by many samples that are also below the threshold (i.e., in the noise-only region). Similar to modified SBS, in order to perform more reliable estimation, it might seem reasonable to continue searching even after one sample above the threshold is found and to look for a specific number of consecutive samples above the threshold. However, since UWB pulses are very narrow in time, it is possible that the first arriving path consists of just one sample. In that case this modification will not work efficiently. Therefore, we propose a different criterion for the modified JBSF algorithm, which involves comparison of the values of all the samples between the test sample and the largest sample against a certain threshold. The index of the test sample is increased at each iteration starting from 1 , until a certain ratio (percentage) of the samples between the test sample and the largest sample are above the threshold. In this way, all samples between the first sample and the strongest sample are considered during the estimation process. Hence, more reliable estimation can be performed compared to JBSF.

Similar to the previous algorithm, the modified JBSF algorithm also starts by finding the largest sample:

$$
N_{\text {max }}=\underset{k \in\left[1, N_{b}\right]}{\arg \max } z[k] .
$$

$N_{\text {test }}$ is set to 1 in the first iteration and all the samples between $N_{\text {test }}$ and $N_{\max }$ are compared with the threshold. In each iteration, $N_{\text {test }}$ is increased by one. The algorithm searches for a specific value of $N_{\text {test }}$ for which a certain ratio of the samples between $N_{\text {test }}$ and $N_{\max }$ are above the 
threshold. Let $\gamma$ denote that certain ratio and $\Delta$ represent the threshold value. Then, the modified JBSF algorithm performs the following test:

$$
\frac{N_{\text {above }}}{N_{\max }-N_{\text {test }}}>\gamma
$$

where

$$
N_{\text {above }}=\sum_{n=N_{\text {test }}}^{N_{\max }} \mathbb{I}\{z[n]>\Delta\}
$$

with $\mathbb{I}$ denoting the indicator function that is equal to one (zero) if the condition holds (does not hold). If the algorithm finds a value of $N_{\text {test }}$ that satisfies the inequality in (5), that value is declared as the index of the first arriving path. Otherwise, $N_{\max }$ is decided to be the index of the first arriving path. The flowchart of the modified JBSF algorithm searching for $90 \%$ of the samples above the threshold (i.e., $\gamma=0.9$ ) is shown in Fig. 3.

\section{Measurement Campaign}

Measurements were collected in the second floor of the Department of Electrical and Electronics Engineering at Bilkent University. The blueprint of the measurement environment is presented in Fig. 4. Two UWB devices were employed in the measurements. One of the devices was kept at a fixed position and used as a reference point. The other device was used as the target point and its position was changed for different sets of measurements. In Fig. 4, the position of the reference point is shown with a red node and different positions of the target point are shown with blue nodes.

Initially LOS measurements were collected in hall 207. The target device was placed at 10 different points, which were chosen in such a way that the distance between the reference device and the target device corresponded to even numbers in the 2-20 meters interval for each point. At each point, 1000 measurements were collected. Then, NLOS measurements were performed by placing the reference device in room 226 and the target device in hall 220 . The wall separating room 226 and hall 220 was $35 \mathrm{~cm}$ thick. Similar to the LOS measurements, the target device was placed at 10 different points and 1000 measurements were collected at each point.

Time Domain's PulsON400 devices were used for the measurements. These devices operate over a band covering the frequencies between $3.1 \mathrm{GHz}$ and $5.3 \mathrm{GHz}$. The sampling frequency is about $16.4 \mathrm{GHz}$. These UWB devices are capable of transmitting different numbers of pulses, and higher signalto-noise ratios (SNRs) can be achieved by employing more pulses. For the measurements, the minimum number of pulses available, which is 128 , is used in order to investigate the most challenging scenarios.

\section{RESUlts}

Performance of the proposed algorithms in Section III is evaluated using the measurements obtained in the measurement environment explained in Section IV. Since the proposed algorithms are threshold based, their performance depends very much on the selection of an appropriate threshold. To perform a fair evaluation, two strategies are employed in this section. First the threshold is set to an ideal value for each

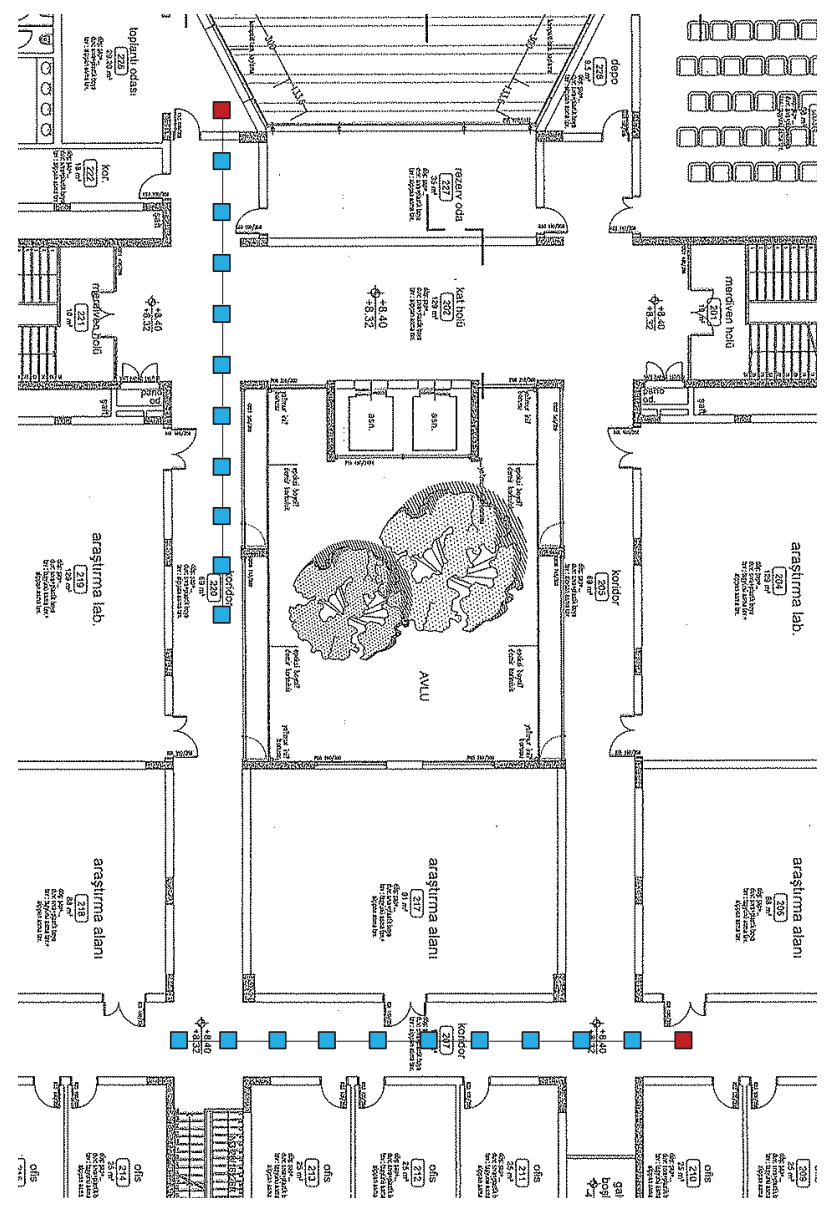

Fig. 4. Blueprint of the measurement environment.

algorithm at each distance. In other words, for each algorithm at each distance, an exhaustive search is performed over all possible threshold values and the one which minimizes the root mean-squared error (RMSE) for that algorithm at that distance is chosen. The second strategy is to set the threshold to an ideal value, which is a fixed value for all distances, for each algorithm. In this case, for each algorithm, an exhaustive search is performed over all possible threshold values and the one which minimizes the RMSE averaged over all distances for that algorithm is chosen.

In Fig. 5, the performance of the standard SBS is compared to that of the modified SBS when two and three consecutive samples below the threshold are searched. Here the ideal values of the thresholds are used at each distance, i.e., the first strategy explained above is employed. In Fig. 6, the RMSEs of the standard SBS algorithm and the modified SBS algorithm are shown for fixed threshold values at each distance, i.e., for the second strategy explained above. It can be observed from Fig. 5 that the modified SBS achieves improvement over the standard algorithm at all distances both in LOS and NLOS situations. It is also noted from Fig. 6 that the modified SBS performs better than the standard algorithm at all distances in the LOS scenario. In the NLOS scenario, the modified algorithm performs better than the standard one at higher ranges, and performs close to the standard algorithm at shorter distances. 

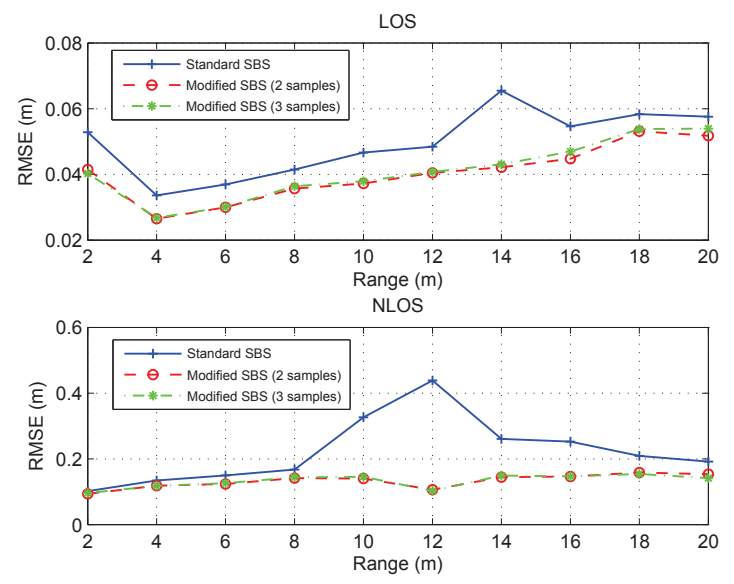

Fig. 5. Estimation errors of standard and modified SBS algorithms for ideal threshold values.
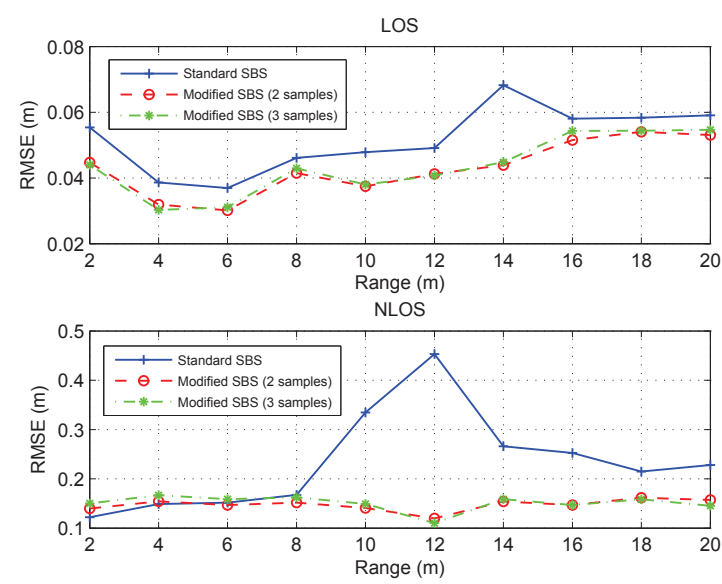

Fig. 6. Estimation errors of standard and modified SBS algorithms for fixed threshold values.

In Fig. 7, the performance of the standard JBSF algorithm is compared to that of the modified JBSF algorithm for different percentage parameters. (Note that the percentage parameter is equal to $100 \gamma \%$, where $\gamma$ is as in (5).) Here the first strategy explained above is used to determine the threshold. It can be observed from the figure that it is possible to achieve a similar performance to the standard algorithm. However, there is no significant improvement. In Fig. 8, the RMSEs of the standard JBSF algorithm and the modified JBSF algorithm are shown for the second strategy explained above. In [9], it was observed that the JBSF algorithm was not stable when fixed parameter values are used. The proposed modification to JBSF is able to solve this problem; that is, for both LOS and NLOS scenarios, the performance of the proposed algorithm does not fluctuate much. It can be observed that the modified JBSF performs better than the standard one when fixed threshold values are used for both LOS and NLOS situations.

It is very important to note that it is not practical to optimize the threshold value for each distance since the distance is
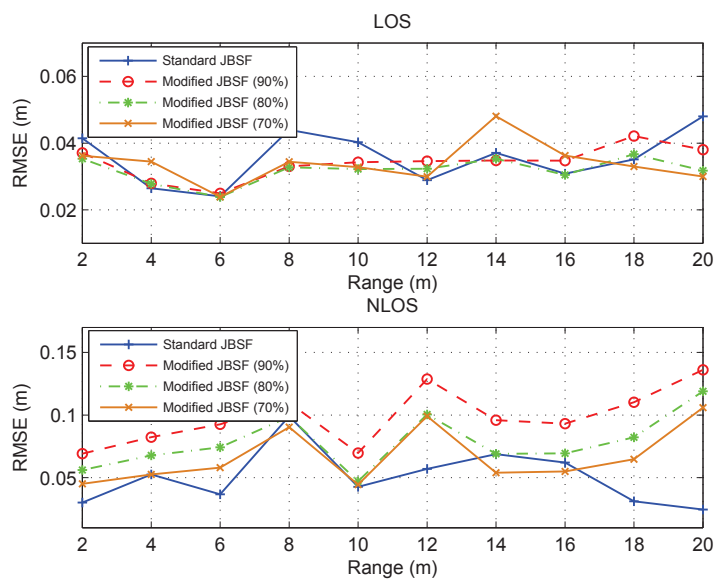

Fig. 7. Estimation errors of standard and modified JBSF algorithms for ideal threshold values.
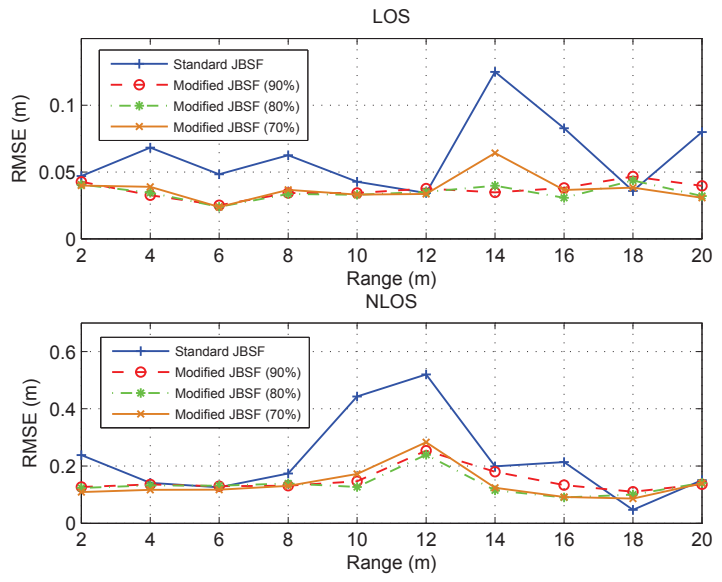

Fig. 8. Estimation errors of standard and modified JBSF algorithms for fixed threshold values.

unknown during the range estimation process. ${ }^{1}$ Therefore, the fixed threshold case considered in Fig. 6 and Fig. 8 corresponds to practical scenarios in which an optimal threshold value is employed for all distances. It is observed that the proposed algorithms provide significant improvements and robustness in this practical scenario.

\section{TheORETICAL Limits}

The range estimation algorithms considered in this study are low-complexity algorithms. Since these algorithms are not optimal, their performance should be compared to theoretical limits to determine if the trade-off between complexity and performance is acceptable or not.

One of the commonly used lower bounds in estimation problems is the CRLB. The CRLB sets a lower bound on the variance of unbiased estimators. It yields a tight bound at high SNR values, whereas at low SNR values the bound may not be very tight. For short range estimation problems, SNR values

${ }^{1}$ Fig. 5 and Fig. 7 are presented for illustrating the best performance that can be achieved at each distance. 
are generally high and therefore the CRLB is a meaningful bound for such applications.

The CRLB for time based ranging can be obtained for both single-path channels and multipath channels [5]. Since it is difficult to know the exact number of paths in the multipath formulation, the single-path formulation is considered here by taking only the first arriving path into account. In fact, this formulation is valid also for multipath channels if the first signal path does not overlap with the other multipath components. (If there is such an overlap, the single-path formulation provides a lower bound on the multipath-formulation.)

For a single path additive white Gaussian noise channel, the CRLB is expressed as

$$
\sqrt{\operatorname{Var}(\hat{d})} \geq \frac{c}{2 \sqrt{2 \pi} \sqrt{S N R} \beta}
$$

where $S N R$ is the signal-to-noise ratio and $\beta$ is the effective signal bandwidth [4]. The value of $\beta$ is equal to $4.28 \mathrm{GHz}$ for the Pulson400 devices used in our measurements.

In the formulation of the CRLB, $S N R$ is the only term depending on the measurements. First of all, for each measurement at each distance, an $S N R$ value is calculated. Since the single-path formulation is considered, only the power of the first arriving path is considered as the signal power. However, in order to have a more reliable result, for each measurement the average power of three consecutive samples starting with the first arriving path is considered as the signal power. On the other hand, the noise power is found as the average power of all the samples coming before the first arriving path. At each distance, 1000 measurements are collected for both LOS and NLOS situations. To reduce the effects of outliers, these measurements are divided into 100 groups which consist of 10 measurements. For each group, an average $S N R$ value is found by averaging the $S N R$ values of 10 measurements in that group. Using this average $S N R$ value, a CRLB value is calculated for each group. Finally, the average of these 100 groups CRLB values is used as the average CRLB value at that distance.

In Fig. 9, the RMSEs of the modified SBS and JBSF algorithms for ideal threshold values are compared to the CRLB for both LOS and NLOS situations. It is observed that the performances of the proposed algorithms are quite close to the bound especially in the LOS situation. Considering the fact that the optimal estimators are quite complicated compared to these algorithms, it can be concluded that these algorithms can be used in practical applications without severe performance degradation.

\section{CONCLUSIONS}

In this study two range estimation techniques have been proposed for real-time UWB positioning applications. These algorithms are inspired from two well-known algorithms; namely, SBS and JBSF. Performances of the proposed algorithms have been tested on real UWB data. It has been observed that the proposed algorithms can perform better than the SBS and JBSF techniques. Also performance of these algorithms has been compared with the theoretical limits and it is observed that the proposed approaches provide good tradeoffs between performance and complexity.
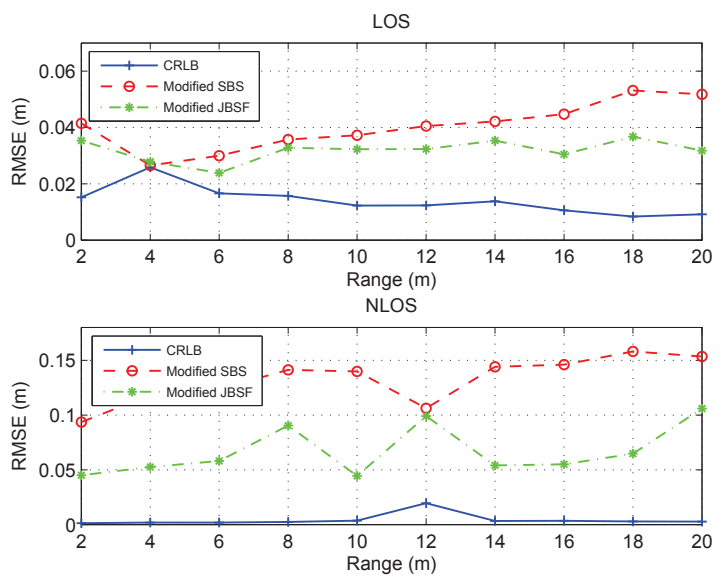

Fig. 9. Comparison of the modified algorithms with CRLB

\section{REFERENCES}

[1] S. Gezici and H. V. Poor, "Position estimation via ultra-wideband signals," Proceedings of the IEEE (Special Issue on UWB Technology and Emerging Applications), vol. 97, no. 2, pp. 386-403, Feb. 2009.

[2] H. Arslan, Z. N. Chen, and M. G. Di Benedetto, Ultra Wideband Wireless Communications, Wiley-Interscience, 2006.

[3] "First report and order 02-48," Federal Communications Commission, Feb. 2002.

[4] Z. Sahinoglu, S. Gezici, and I. Guvenc, Ultra-Wideband Positioning Systems: Theoretical Limits, Ranging Algorithms, and Protocols, Cambridge, U.K.: Cambridge Univ. Press, 2008.

[5] S. Gezici, Z. Tian, G. B. Giannakis, H. Kobayashi, A. F. Molisch, H. V. Poor, and Z. Sahinoglu, "Localization via ultra-wideband radios: A look at positioning aspects for future sensor networks," IEEE Signal Process. Mag., vol. 22, pp. 7084, July 2005.

[6] S. J. Ingram, D. Harmer and M. Quinlan, "Ultra-wideband indoor positioning systems and their use in emergencies," in Proc. IEEE Position Location and Navigation Symp. (PLANS), pp. 706715, Monterey, CA, Apr. 2004.

[7] C. Falsi, D. Dardari, L. Mucchi, and M. Z.Win, "Time of arrival estimation for UWB localizers in realistic environments," EURASIP J. Applied Sig. Processing, vol. 2006, Article ID32082, 13 pp. 2006. doi:10,1155/ASP/2006/32082

[8] I. Guvenc and Z. Sahinoglu, "Threshold-based TOA estimation for impulse radio UWB systems," in Proc. IEEE Int. Conf. UWB (ICU), pp. 420-425, Zurich, Switzerland, Sep. 2005.

[9] H. Soganci, S. Gezici and A. S. Tan, "Comparison of low complexity UWB range estimators based on experimental data," IEEE Signal Processing and Communications Applications Conference (SIU 2012), Fethiye, Turkey, April 18-20, 2012.

[10] H. Soganci, S. Gezici and H. V. Poor, "Accurate positioning in ultrawideband systems," IEEE Wireless Communications, pp. 19-27, April 2011.

[11] Z. Sahinoglu and S. Gezici, "Ranging in the IEEE 802.15.4a standard," IEEE Wireless and Microwave Technology Conference (WAMICON 2006), Clearwater, FL, Dec. 4-5, 2006.

[12] R. A. Scholtz and J. Y. Lee, "Problems in modeling UWB channels," in Proc. IEEE Asilomar Conf. Signals, Syst. Computers, vol. 1, pp. 706-711, Monterey, CA, Nov. 2002.

[13] D. Dardari and M. Z. Win, "Threshold-based time-of-arrival estimators in UWB dense multipath channels," in Proc. IEEE Int. Conf. Commun. (ICC), vol. 10, pp. 4723-4728, Istanbul, Turkey, June 2006. 